Conclusions Although recommendations are being followed, there is still place for antibiotic therapy in RSV infection. The need for antibiotic cannot be easily predicted upon traditionally used inflammatory markers. Due to prolonged hospital stay, there is strong need for minimizing antibiotic use, and more precise clinical tools to assess the risk of antibiotic.

\section{GP196 THE USE OF A RAPID ANTIGEN DETECTION TEST FOR BETA HAEMOLYTIC GROUP A STREPTOCOCCUS TO AID THE MANAGEMENT OF PHARYNGITIS AND TONSILLITIS IN AN IRISH TERTIARY PAEDIATRIC EMERGENCY DEPARTMENT}

Caroline Fox*, Patrick Fitzpatrick. Children's University Hospital, Temple Street, Dublin, Ireland

\subsection{6/archdischild-2019-epa.256}

Background Acute sore throat is a common presentation to the Emergency Department (ED). Rapid-antigen detection testing (RADT) is used in our department to aid diagnosis of Group A streptococcus (GAS) as the cause of pharyngitis/tonsillitis as an adjunct to clinical assessment. Our aims were to assess use of RADT in management and treatment of pharyngitis/tonsillitis in the ED and compare our practice with current NICE guidelines.

Methods This was a prospective study which took place at the Children's University Hospital Dublin in 2018. A proforma was created and doctors were asked to complete this for children who had a RADT for GAS. Data collected included patient age, history, examination findings, rapid-antigen swab result, use of throat swab culture, use of antibiotics. The modified Centor score (MCS) was then calculated.

Results Data collected on 102 patients. 1 patient excluded as data form incomplete. $16(15.8 \%)$ patients had low MCS of 0,1 or 2 . Of these, 1 patient was RADT positive and treated with antibiotics. $85(84.2 \%)$ patients had high MCS of 3, 4 or $5.26(30.6 \%)$ were RADT positive and were treated with antibiotics. $59(69.4 \%)$ were RADT negative -6 were treated with antibiotics.

Of the 74 patients with a negative RADT, 20 of these had a throat culture sent. $25 \%$ had GAS positive culture.

$42 / 101$ patients were treated with antibiotics. 27 of these were RADT positive. Of the RADT negative patients, 8 were treated with antibiotics by the ED physician for pharyngitis/ tonsillitis, 3 were treated for other diagnoses, 4 had antibiotics continued that were started by a primary care physician. Of the 27 children with a positive RADT swab, 92.5\% had a MCS of 4 or 5 .

Conclusions NICE guidelines suggest no benefit of RADT testing over clinical scores alone. The low incidence of RADT positivity in the low risk MCS group (MCS 0,1 or 2) suggests we can safely not test and not offer antibiotics to these children. In the high MCS group (MCS 3, 4 or 5), only $37.6 \%$ of patients had antibiotics started by the ED physician suggesting that RADT may have a role in reducing the number of patients treated with antibiotics.

A formal guideline will be created for use in our ED. In communities where the incidence of rheumatic fever is low, a balance must be made between reducing symptoms by a modest amount and the emerging issue of antimicrobial resistance. COMMUNITY

Ariel Halperin, Yehudith Shindler, Alexandra Gleyzer, Yoram Ben Yehuda, Eli Somekh* Mayenei, Hayeshuah, Israel

\subsection{6/archdischild-2019-epa.257}

Introduction Rates of community acquired methicillin resistant staphylococcus aureus (MRSA) in Israel is quite low and estimated at the range of $3 \%$ out of staphylococcus aureus isolates.

This survey has been undertaken due to clinical impression of significant rise at the rates of MRSA isolates during the last few years in a closed community in Israel.

Methods All community acquired staphylococcus aureus isoltes from children referred to Mayenei Hayeshuah Hospital in Bnei Brak Israel during the years 2015-2018 were analyzed. This hospital serves a closed Ultraorthodox Jewish community characterized by crowdedness.

Results A total of 201 isolates were reviewed. Most isolates (163) were from skin and soft tissue specimens and the rest were from normally sterile fluids, urine and ear specimens.

The rates of MRSA isolates out of all staphylococcal isolates were $14 \%$.

Most MRSA isolated were from the skin and soft tissue while none of the isolated from normally sterile fluid fluids grew out MRSA.

During the study years there was a dramatic rise at the rates of MRSA from 4\% in 2015 to $23 \%$ in 2018 .

Children with MRSA infections were younger than those with methicillin sensitive staphylococcus aureus (MSSA) infection (mean ages were 2.9 years and 5.9 years in MRSA vs. MSSA infected children respectively, $\mathrm{p}<0.001$ ).

Clindamycin inducible resistance was detected in $44 \%$ of MSSA isolates and in 7\% of MRSA isolates.

Trimethoprim/sulfamethoxazole resistant was observed in $2 \%$ of MSSA and in 7\% of MRSA isolates.

Conclusions These findings demonstrates the ability of MRSA clones to spread rapidly especially in a closed and crowded community.

Our findings also indicate that clindamycin is not an appropriate antibiotic for empiric treatment of staphylococcal infection unless administered with another anti staphylococcal agent.

In addition, the increased rate of trimethoprim/sulfamethoxazole resistant is worrisome and should be closely monitored.

\section{GP198 SOMETHING WICKED THIS WAY COMES. THE FIRST PAEDIATRIC CASES OF ENTEROVIRUS D68-ASSOCIATED ACUTE FLACCID MYELITIS IN IRELAND}

${ }^{1}$ Elaine Kennedy*, ${ }^{2}$ Cillian deGascun, ${ }^{2}$ Allison Waters, ${ }^{3}$ Richard Drew, ${ }^{1,4}$ Karina Butler, ${ }^{1,4}$ Patrick Gavin. ${ }^{1}$ Rainbow Paediatric Infectious Diseases, Temple Street Children's University Hospital, Dublin, Ireland; ${ }^{2}$ National Virus Reference Laboratory, University College Dublin, Dublin, Ireland; ${ }^{3}$ Irish Meningitis and Sepsis Reference Laboratory, Temple Street Children's University Hospital, Dublin, Ireland; ${ }^{4}$ Rainbow Paediatric Infectious Diseases, Our Lady's Children's Hospital Crumlin, Dublin, Ireland

\subsection{6/archdischild-2019-epa.258}

Introduction Enterovirus (EV) D68 is a non-polio enterovirus closely related to rhinovirus. In contrast to the majority of EV, EV D68 is primarily a respiratory virus. However, like polio and EV D71, EV D68 displays neurotropism and the 
ability to cause acute flaccid myelitis. Acute flaccid myelitis (AFM) refers to acute flaccid limb weakness with predominantly gray matter spinal cord lesions on imaging or evidence of anterior horn cell damage on electrodiagnostic studies. In 2014 , in the midst of a large outbreak of EV D68-associated respiratory disease in the United States, clusters of AFM were noted in children. Enhanced nationwide surveillance ultimately identified 120 cases of AFM. Enterovirus D68 was the most common virus detected in these patients. Full recovery was rare (5\%). More recently, a 2019 report describes 29-cases of EV D68-associated AFM diagnosed in 12 European countries in 2016. We present the first cases of EV D68-associated AFM in Ireland to date.

Clinical cases In June 2016, a previously well 2-year 9 month old girl presented with a one-week history of cough, fever and vomiting. Thirty-six hours later, respiratory status worsened and she developed AFM and bulbar palsy necessitating intubation and ventilation. CSF analysis demonstrated a lymphocytic pleocytosis. In October 2018, a 4-year old boy, with a background of acute lymphoblastic leukaemia diagnosed 17 months previously, presented with fever, worsening cough and pancytopenia. Three-days later, he required intubation and ventilation after a respiratory arrest. On weaning sedation, eight-days later, he displayed clinical signs of AFM. Both children had nasopharyngeal aspirates (NPA) PCR positive for rhinovirus/enterovirus, and stool/rectal swabs PCR positive for EV. CSF was PCR negative for EV, HPE, HSV, HHV-6, VZV and adenovirus. MRI showed extensive abnormal signal predominantly of white matter in the cervico-thoracic cord. Electromyography was indicative of anterior horn cell involvement. Both children required prolonged ICU and hospital stays, have severe residual neurologic deficits with flaccid quadriparesis, tracheostomy and require ongoing ventilatory support.

Results Subsequent subtyping of EV isolates from both children was positive for EV D68. Additional testing of 800 rhinovirus/enterovirus-positive NPA specimens submitted to the National Virus Reference Laboratory (NVRL) from August to October 2018 detected 30 (3.75\%) EV D68-positives.

Conclusion This report of two-children with rapidly progressive AFM following prodromal febrile respiratory or gastrointestinal illness in association with EV D68 infection and recent results of enhanced European and local surveillance suggests that EV D68 AFM has arrived in Ireland.

\section{GP199 CELL RATIOS IN TRAUMATIC CEREBROSPINAL FLUID. DO THEY HAVE PREDICTIVE VALUE FOR MENINGITIS?}

Ali Raba*, Jean Donnelly. Our Lady's Children's Hospital, Crumlin, Dublin, Ireland

\subsection{6/archdischild-2019-epa.259}

Background and aims A cerebrospinal fluid (CSF) red to white cell correction ratio of $500: 1$ is often quoted as a preliminary result for determining potential positive/negative CSF cultures. The aim of this study is to examine the diagnostic utility of correcting white blood cells (WBC) counts in CSF based on 500:1 ratio and to evaluate this adjustment in identifying bacterial meningitis prior to final culture in infants.

Method We performed a cohort study on children younger than 3 months who admitted to Our Lady's Children's Hospital, Crumlin, with suspected sepsis from the $1^{\text {st }}$ October 2017 to the $05^{\text {th }}$ October 2018. CSF WBC counts were adjusted downward for traumatic lumbar punctures (RBCs > $500 / \mathrm{mm} 3)$ using white to red cells $(500: 1)$ ratio. We calculated sensitivity, specificity, positive productive value (PPV) and negative productive value (NPV) of unadjusted and adjusted WBC count in traumatic CSF for predicting bacterial meningitis.

Result A total of 117 infants (age: $39.4 \pm 21.7$ days, male: 85 (72.6\%), traumatic lumber puncture: $35(29.9 \%)$ ) were enrolled in this study. Among 117 infants, 3 (2.6\%) had positive CSF cultures for bacterial meningitis, 28 (23.9\%) had viral meningitis. Compared with the unadjusted cerebrospinal fluid WBC count, the adjusted one had similar sensitivity, PPV and NPV for bacterial meningitis. NPV and PPV of adjusted CSF WBC count were $100 \%$ and $14.2 \%$, respectively (sensitivity $100 \%$, specificity $82 \%$ ). While NPV and PPV of unadjusted one were $100 \%$ and $5.8 \%$, respectively (sensitivity 100\%, specificity $52 \%)$.

Conclusion Adjusted WBC counts in CSF have no advantage over uncorrected counts for predicting bacterial meningitis. All available clinical and laboratory data should be used when deciding whether to institute treatment in an infant with suspected meningitis and bloody CSF.

\section{GP200 IS THE INFLUENZA IN NEONATES A DANGEROUS INFECTION? - OWN OBSERVATIONS}

1,2Edyta Zawłocka*, 1,2 Małgorzata Czajkowska, 1,2 August Wrotek, 1,2Teresa Jackowska. ${ }^{1}$ Department of Pediatrics, the Medical Centre of Postgraduate Education, Marymoncka 99/ 103, Warsaw, Poland; '2Department of Pediatrics, Bielanski Hospital, Cegłowska 80, Warsaw, Poland

\subsection{6/archdischild-2019-epa.260}

Background Influenza is a viral infectious disease affecting every age group, including neonates and infants.

Aim To analyze the frequency, clinical signs and course of influenza in neonates hospitalized during three influenza epidemic seasons.

Material and Methods 12 neonates were hospitalized due to influenza in Department of Pediatrics in three influenza epidemic seasons. Rapid Influenza Diagnostic Tests (RIDTs) and/ or Real Time-Polymerase Chains Reaction Tests (RT-PCR) were performed in patients with a clinical suspicion of influenza.

Results From September 2015 to August 2018 we hospitalized 294 patients with influenza. The youngest patient was 12 days old, the oldest - 17 years 3 months. 4.1\% (12/294) were neonates. The most common reason for hospital referral was fever $(58.3 \%$; 7/12). Other observe symptoms were: coryza $(58.3 \% ; 7 / 12)$, change of behavior $(58.3 \% ; 7 / 12)$, cough $(50 \% ; 6 / 12)$, dyspnea $(40 \% ; 5 / 12)$ and apneas $(8.3 \% ; 1 / 12)$. RIDTs were performed in seven $(58.3 \%)$ of neonates, but positive results were only in 2 cases (16\%). The final diagnosis was made using RT-PCR tests. $83.3 \%(10 / 12)$ of neonates were diagnosed with influenza A, and 1.7\% (2/12) influenza B. Complications occurred in eight neonates. $75 \%(9 / 12)$ patients had contact with the infection at home. None of the families were influenza-vaccinated before the season. The average time of hospitalization was 10.2 days (from 6 to 15 days).

Conclusion The course of influenza in neonates can be severe. RIDTs seem not very useful in diagnose.

This study was supported by CMKP. Grant number 5011--20-19-18 